\author{
ANDREAS J. STYLIANIDES ${ }^{1}$, GABRIEL J. STYLIANIDES ${ }^{1}$ and \\ GEORGE N. PHILIPPOU ${ }^{2}$
}

\title{
UNDERGRADUATE STUDENTS' UNDERSTANDING OF THE CONTRAPOSITION EQUIVALENCE RULE IN SYMBOLIC AND VERBAL CONTEXTS
}

\begin{abstract}
Literature suggests that the type of context wherein a task is placed relates to students' performance and solution strategies. In the particular domain of logical thinking, there is the belief that students have less difficulty reasoning in verbal than in logically equivalent symbolic tasks. Thus far, this belief has remained relatively unexplored in the domain of teaching and learning of mathematics, and has not been examined with respect to students' major field of study. In this study, we examined the performance of 95 senior undergraduate mathematics and education majors in symbolic and verbal tasks about the contraposition equivalence rule. The selection of two different groups of participants allowed for the examination of the hypothesis that students' major may influence the relation between their performance in tasks about contraposition and the context (symbolic/verbal) wherein this is placed. The selection of contraposition equivalence rule also addressed a gap in the body of research on undergraduate students' understanding of proof by contraposition. The analysis was based on written responses of all participants to specially developed tasks and on semi-structured interviews with 11 subjects. The findings showed different variations in the performance of each of the two groups in the two contexts. While education majors performed significantly better in the verbal than in the symbolic tasks, mathematics majors' performance showed only modest variations. The results call for both major- and context- specific considerations of students' understanding of logical principles, and reveal the complexity of the system of factors that influence students' logical thinking.
\end{abstract}

KEY WORDS: contraposition equivalence rule, logic, proof, reasoning, reasoning domains, undergraduate education

\section{INTRODUCTION}

Reasoning and proof are defining features of mathematics and, in present day reform recommendations, are considered fundamental aspects of instructional programs in all grade levels (NCTM, 2000; Yackel and Hanna, 2003). Numerous studies have examined students' reasoning skills and understanding of proof in elementary (e.g., Ball and Bass, 2000, 2003; Carpenter et al., 2003; Maher and Martino, 1996; Reid, 2002; Zack, 1997), secondary (e.g., Chazan, 1993; Coe and Ruthven, 1994; Edwards, 1999; Fischbein and Kedem, 1982; Healy and Hoyles, 2000; Hoyles and Küchemann, 2002; Knuth et al., 2002; Porteous, 1990; Silver and Carpenter, 
1989; Silver et al., 2000), and undergraduate level (e.g., Gardiner and Moreira, 1999; Harel and Sowder, 1998; Martin and Harel, 1989; Moore, 1994; Recio and Godino, 2002; Schoenfeld, 1986). Despite the abundance of research on students' reasoning and understanding of proof, this body of research has tended not to focus on specific types of proof.

Some researchers studied students' understanding of particular types of proof, such as proof by counterexample and inductive/empirical proof (Epp, 1998; Galbraith, 1981; Goetting, 1995; Harel and Sowder, 1998; Morris, 2002; Porteous, 1990; Silver, 1998; Simon and Blume, 1996; Stylianides et al., 2002; Szombathelyi and Szarvas, 1998), proof by contradiction (Epp, 1998; Goetting, 1995; Knuth, 1999; Tall, 1979), proof by mathematical induction (Dubinsky, 1986, 1990; Fischbein et al., 1982; Knuth, 1999; Markel, 1994; Movshovitz-Hadar, 1993), and proof by contraposition (Goetting, 1995), but proof by contraposition is, perhaps, the method of proof that has attracted the least research attention thus far.

Goetting's (1995) dissertation study about undergraduate students' understanding of different kinds of proof was the only study we have been able to locate that examined students' understanding of proof by contraposition. Goetting conducted semi-structured interviews with 40 undergraduate students; the sample consisted of 11 pre-service elementary teachers, 16 pre-service secondary mathematics teachers, and 13 advanced students primarily, but not exclusively, majoring in computer science, mathematics, or engineering. The types of proof that attracted Goetting's attention, other than proof by contraposition, included inductive and deductive arguments in support of generalizations, counterexamples to generalizations, and proof by contradiction. It is worth noting that the elementary majors along with five other students were excluded from the part of the study that investigated students' understanding of both proof by contradiction and proof by contraposition. The remaining 24 students were asked to react to a given proof by contraposition for the following statement: "For any integer $b$, if 2 is a factor of $b^{2}$, then 2 is a factor of $b$ ". Goetting notes that only one student seemed comfortably acquainted with the contraposition equivalence rule used in the proof; this student said that she would explain the rule to a questioning student using truth tables. Evaluating the findings of her study with regard to proof by contraposition, Goetting notes that many students had difficulties distinguishing proof by contraposition from proof by contradiction. Some of these students confused what was to be shown with what could be considered as given. Several other students thought necessary to include in the given proof an extra argument for $b$ being even. Some students did not even consider proof by contraposition as having any bearing on the specific statement that was proved with this 
particular method, and others "seemed to be wary of the validity of this 'backwards' argument" used in the proof (Goetting, 1995, p. 142).

The first focus of this paper is to extend what we currently know about undergraduate students' understanding of proof by contraposition, which is an important method in proving universal conditionals of the form $\forall x$ $\in D$, if $P(x)$ then $Q(x)$. More precisely, we aim to explore more broadly undergraduate students' understanding of the contraposition equivalence rule ( $p \Rightarrow q \equiv \sim q \Rightarrow \sim p$ ), which is fundamental to proof by contraposition.

The context in which contraposition equivalence rule was placed varied among studies in the mathematics education literature. In several cases (O'Brien et al., 1971; O'Brien, 1972, 1975) contraposition equivalence rule was placed in verbal context - the setting of language statements expressed in words -, while in some others (Goetting, 1995) contraposition equivalence rule was placed in symbolic context - the setting of mathematical notation ${ }^{1}$. The distinction between verbal and symbolic contexts is hypothesized, in this study, to be critical in students' ability to reason about contraposition equivalence rule; the exploration of this assumption is the second focus of this paper. The type of context wherein a task is placed - also referred to as the reasoning domain - has been found to relate to students' performance and solution strategies across grade levels. A significant body of research investigates mathematics problem solving and reasoning across different contexts. In particular, Epp (1998) has argued that introducing undergraduate students to logical principles via English language statements can bring about better results than using the notation of symbolic logic.

Morris (2002), drawing on numerous studies that examined adolescents' and adults' logical competence in distinguishing between necessary and indeterminate forms of argument, makes the point that there is a discrepancy between performance in mathematics (symbolic, in our terms) and other reasoning domains, such as the verbal syllogistic (verbal, in our terms):

In several types of tasks, the majority of adolescents and adults have shown that they can distinguish between necessary and indeterminate forms of argument (e.g., Braine and Rumain, 1983; Markovits and Vachon, 1989; Moshman and Franks, 1986; Scholnick and Wing, 1988; Ward and Overton, 1990) .... When the content of a logical task is mathematical, however, the majority of adolescents and adults appeared to fail to make this distinction (e.g., Lee and Wheeler, 1987; Martin and Harel, 1989; Morris and Sloutsky, 1998; Williams, 1979). It is unclear what factors could account for the discrepancy between performance in mathematics and other reasoning domains.

(Morris, 2002, p. 80)

Morris suggests that adolescents and adults face more difficulties in distinguishing between necessary and indeterminate forms of argument when 
these kinds of argument are placed in symbolic rather than in verbal contexts. While the argument that certain kinds of logical competence are lower in a symbolic reasoning domain than in other reasoning domains seems plausible, it is worth noting that Morris's statement is mostly based on studies that are not specific to the teaching and learning of mathematics, and that have not examined their subjects' logical competence in both symbolic and verbal contexts. Also, the participants of these studies have inhomogeneous characteristics; this fact imposes further constraints on any attempt to make an argument that spans different studies. In this paper, we claim that the possible association between reasoning domain - and in, particular, symbolic and verbal contexts - and students' understanding of logical principles central to mathematical argumentation can systematically be investigated with comparison of the performances of the same group of participants in these two contexts. The present study contributes to this relatively unexplored domain of mathematics education research by examining the performances of a group of undergraduate students in tasks that involve application of the contraposition equivalence rule in both symbolic and verbal contexts ${ }^{2}$. Contraposition equivalence rule is particularly appropriate for this kind of investigation, for it is a logical operation that can easily be examined in both verbal and symbolic contexts; this characteristic of contraposition equivalence rule provided further motivation for using it in this study.

Finally, in the present study we also hypothesize that students' major field of study may influence the way students' performance is associated with the context wherein contraposition equivalence rule is placed. This hypothesis is grounded on the premise that different programs of study may promote to different extents and in varying ways students' ability to reason in symbolic and verbal contexts. Based on this, the participants of this study were selected to be senior students majoring in one of two different fields - education and mathematics. Students' major is believed to be a possible factor influencing the relation between performance and context, mainly due to the different learning experiences students acquire as a result of their contact with the collegiate curriculum of their program of study. The selection of these two groups of participants is motivated by the presumably different emphases placed by the education and mathematics collegiate curricula on symbolic and verbal reasoning domains; the mathematics program of study is considered to promote more the former, whereas the education is believed to promote more the latter. Despite these different emphases, if we accept the hypothesis that logical competence in contraposition is, in general, lower in symbolic context than in verbal context, then both groups of participants are expected to perform better in 
reasoning about contraposition in a verbal rather than in a symbolic context. In this sense, the case of mathematics majors attracts special interest, for, as we noted earlier, it is plausible to assume that the mathematics collegiate curriculum emphasizes more reasoning in the symbolic rather than in the verbal context.

To sum up, the research questions we investigate in this paper span the following themes: students' understanding of contraposition, the relation between students' performance in tasks about contraposition equivalence rule and the context (symbolic/verbal) in which this rule is placed, and the role of students' major (education/mathematics) in this relation. Specifically, we examine the following three (interrelated) research questions:

1. What is students' understanding of contraposition equivalence rule (placed in both symbolic and verbal contexts)?

2. How does students' performance in tasks about contraposition equivalence rule relate to the context (symbolic/verbal) wherein this rule is placed?

3. What is the relation between students' major (education/mathematics) and their performance in tasks about the contraposition equivalence rule placed in different contexts (symbolic/verbal)?

\section{Methods}

\subsection{Participants}

The subjects are senior undergraduate students of the University of Cyprus majoring either in mathematics or in education. The sample consists of 95 students -70 education majors [EM] and 25 mathematics majors [MM] who participated in a larger study which aimed to elicit and compare the conceptions of different types of proof held by the seniors of the Department of Education and the Department of Mathematics. Given our focus on students' major as a possible factor influencing the way students' performance is associated with the context wherein contraposition equivalence rule is placed, we considered important to choose a sample representative of the senior students of the two departments. The EM that participated in the study constituted the $50 \%$ of the 2000-01 seniors of the Department of Education; all of them were taking one particular class during the Fall 2000 semester to which they were allocated randomly. The $25 \mathrm{MM}$ were all the seniors of the Department of Mathematics in the academic year 2000-01.

It is worth noting that, with only very few exceptions, those graduating from the Department of Education become elementary school teachers 
(grades K to 6). Most of the graduates of the Department of Mathematics eventually become secondary school teachers of mathematics (grades 7 to 12). In the public school system of the Republic of Cyprus, mathematics majors are the only graduates qualified to teach mathematics in secondary schools. In this sense, the participants of this study may also be considered prospective elementary school teachers and potential secondary school teachers of mathematics. The two groups of students participating in the study (namely, education and mathematics majors) were not selected, however, because of their professional aspirations (or opportunities); rather, they were selected because of their different field-of-study concentrations and the presumably different degrees of emphases these concentrations place on verbal and symbolic reasoning domains.

Another important issue to be mentioned is that, contrary to what might be expected by readers from other countries, the Department of Education has been the most competitive department of the University of Cyprus to be admitted to. Many different reasons - most notably, guaranteed teaching positions, high salary, and fringe benefits - influence Cypriot students to major in education and result in high admission standards (Papanastasiou and Papanastasiou, 1997, 1998) ${ }^{3}$. The Department of Mathematics also ranks high in the preferences of incoming students.

Finally, it should be noted that the EM at the University of Cyprus take several mathematics courses that emphasize logical thinking and ask for proofs. Specifically, the program of study at the Department of Education requires that all students take the following courses relevant to mathematics: (a) Two courses on foundations and fundamental concepts of mathematics ${ }^{4}$; (b) One introductory course on statistical methods and probability; and (c) One course on mathematics teaching and learning. These courses provide a fair amount of mathematical knowledge about different types of proof (including proof by contraposition) that allowed us to include this group of students in our study. The MM at the University of Cyprus are not required to take any courses in the teaching and learning of mathematics; their preparation focuses primarily on mathematical content and abstract thinking.

\subsection{Data and procedure}

All 95 participants responded to a specially designed test that included items on different types of proof, such as empirical/inductive proof, proof by counterexample, proof by contradiction, proof by mathematical induction, and proof by contraposition. The data from the test were supplemented by interviews with 11 subjects, eight education and three mathematics majors. 
TABLE I

Description of the three test items

\begin{tabular}{|c|c|c|}
\hline Test Item & \multicolumn{2}{|l|}{ Description } \\
\hline Question 1 & \multicolumn{2}{|c|}{$\begin{array}{l}\text { Consider the following statement: } \\
\qquad \text { If } x^{2} \neq y^{2} \text { then } x \neq y \text { (where } x, y \in \mathbf{N} \text { ). } \\
\text { Study carefully the following proof for the above statement. } \\
\text { Proof: } \\
\text { If } x=y \Rightarrow x^{2}=y^{2} \\
\text { Hence, the statement is true. } \\
\text { Choose the best response for the above proof: } \\
\text { 1. The proof is false. } \\
\text { 2. The proof shows that the statement is always true. } \\
\text { 3. The proof shows that the statement is true in some cases. } \\
\text { 4. I have no opinion. }\end{array}$} \\
\hline \multirow[t]{4}{*}{$\begin{array}{l}\text { Questions } 2 \\
\text { and } 3\end{array}$} & \multicolumn{2}{|c|}{$\begin{array}{l}\text { Study the statements and the conclusion that follows them. Circle the } \\
\text { response that represents better what you think about each conclusion, } \\
\text { and explain your thinking. }\end{array}$} \\
\hline & Question 2 & Question 3 \\
\hline & $\begin{array}{l}\text { Statements: } \\
\text { - If Costas suffered from pneu- } \\
\text { monia, he would have high fever. } \\
\text { - Costas does not have high fever. } \\
\text { Conclusion: } \\
\text { Costas definitely does not suffer } \\
\text { from pneumonia. }\end{array}$ & $\begin{array}{l}\text { Statements: } \\
\text { - If the car doesn't have fuel, it } \\
\text { will not move. } \\
\text { - The car has fuel. } \\
\text { Conclusion: } \\
\text { The car will definitely move. }\end{array}$ \\
\hline & $\begin{array}{l}\text { 1. The conclusion is correct. } \\
\text { 2. The conclusion is wrong. } \\
\text { 3. I do not have enough clues to } \\
\text { decide. }\end{array}$ & $\begin{array}{l}\text { 1. The conclusion is correct. } \\
\text { 2. The conclusion is wrong. } \\
\text { 3. I do not have enough clues to } \\
\text { decide. }\end{array}$ \\
\hline
\end{tabular}

Table I shows the three test items that we report on in this study ${ }^{5}$. The first item (Q1) included a simple proof by contraposition, the validity of which was to be judged by the students (the proof was correct, so the right answer was choice ' 2 '). The item was designed so that the proof was totally dependent on the contraposition equivalence rule. In other words, students were expected to say that the proof was correct only if they believed that the given implication statement and its contrapositive were equivalent. Assuming that one accepts the contraposition equivalence rule, the statement 'If $x=y \Rightarrow x^{2}=y^{2}$ ' is taken to be a (correct) proof for the given statement (that is, a convincing argument about the validity of the given statement), because the truth of the proposition 'If $x=y \Rightarrow x^{2}=y^{2}$ ' is readily acceptable 
by anyone with basic mathematical preparation. The statement that was to be proved, on the other hand, is by far less obvious - this less obvious character is what makes the search for a proof meaningful. Finally, the design of a proof totally dependent on the contraposition equivalence rule helped control other factors that would probably emerge in a more lengthy and complicated proof. Goetting (1995) reports, for example, that some students rejected a proof by contradiction because they were unable to follow an intermediate algebraic step.

The second item (Q2) included two statements and a conclusion. The subjects were asked to evaluate the validity of the conclusion, given the two statements, and explain their thinking. The conclusion was correct (i.e., the right choice was ' 1 ') and was based on the contraposition equivalence rule $p \Rightarrow q \equiv \sim q \Rightarrow \sim p$. The third item (Q3) had the same structure as the previous one. This time, however, the conclusion was wrong (i.e., the right answer was ' 2 ') and was based on the wrong equivalence $p \Rightarrow q$ $\equiv \sim p \Rightarrow \sim q$. The word 'definitely' in the conclusions of the two questions was making clear that the implications were intended to carry necessity. As it is noted by Durand-Guerrier (2003), there is generally a confusion shared among students and teachers on whether an implication in mathematics expresses necessity; the design of the tasks excluded this possible confusion, the investigation of which was not central to our goals.

Q1 and Q2 were intended to test, respectively, students' conceptualization of contraposition in symbolic and verbal contexts. Q3 was included for a more integrated investigation of students' understanding of contraposition, for it was testing students' understanding of the other possible 'logical equivalence' that could be formed with the conditional sentence $p \Rightarrow q$, and the propositions $\sim p$ and $\sim q$ when connected with the implication symbol; in this sense, Q3 can also be considered as a task 'about contraposition'. The inclusion of Q3 in the test was motivated by research findings indicating that students often believe that an implication statement $p \Rightarrow q$ is equivalent to its inverse $(\sim p \Rightarrow \sim q)$ rather than to its contrapositive $(\sim q \Rightarrow \sim p)$ (Goetting, 1995; Knuth, 1999, 2002). Q3 provided, in addition, another opportunity to examine students' performance in a 'logical equivalence' placed in verbal context.

\subsection{Interviews}

The interviews were conducted with students whose responses to the test items seemed to be of special interest to the research questions of the larger study; in this sense, the 11 subjects selected for an interview constituted a purposeful sample (Patton, 1990). Special interest was defined primarily in terms of exploring further common student understandings, eliciting 
explanations to responses at which students omitted to provide one, and clarifying infrequent responses. Student identification was possible, for students were asked to write their names on the test - students responded positively to this request with only very few exceptions. The interviews lasted approximately 35 minutes each, were audio taped and fully transcribed. They were also carried out in the presence of two interviewers (the first two authors), who tried to supplement each other in taking notes and asking questions that would elicit students' understanding. In this report, we draw on data relevant to the research questions of the present paper.

The interview segments from which we draw on in this paper were intended to explore students' understanding of contraposition - the different ways of thinking reflected in their written explanations in the test items and the rationale that guided their responses to the multiple-choice questions. In preparing for the interviews, the interviewers would note aspects of students' responses that were of special interest, as explained previously. The interviewers worked collaboratively to prepare from two to five questions each time, with relevant probes for use as necessary. Issues of interest to our research questions that emerged from the discussion were explored further; to preserve space for this kind of examination the interviews were given a semi-structured character (Merriam, 1988). A topic that was pursued in almost all interviews, irrespective of students' written explanations, was associated with students' understanding of the common logical structure underlying Q1 and Q2 in the test - that is, the fact that both problems were based on the same logical equivalence. In most of the interviews, the discussion about students' understanding of contraposition equivalence rule started with a focus on a particular student response in the test and probing the student to elaborate on that. For example, a typical initial question was: "In question 2, you said that the conclusion is false. Could you elaborate on how you thought about it?"

\subsection{Analysis}

The statistical analysis was performed by using the statistical package SPSS. First, frequencies, percentages, and crosstabs tables of major by Q1, Q2, and Q3 were obtained. The descriptive statistics gave a sense of students' understanding of contraposition, for they also provided information about the distribution of wrong answers. Variables Q1 through Q3 were then recoded into PQ1 through PQ3, respectively, which represented students' performance in the three questions (dichotomous scale ' 0 ' and ' 1 '). $t$-tests were run to test the differences between students' (EM and MM considered together) performances in Q1 and Q2, Q1 and Q3, and Q2 and $\mathrm{Q} 3$; these tests helped explore the relation between students' performances 
in tasks about contraposition and the context in which this is placed. To explore the role of students' major, chi-square tests of Independence were carried out to examine the relationship between major and performance in each of the three questions. Furthermore, to examine the influence of students' major on the way their performance is associated to the context wherein contraposition is placed $t$-tests were run to test the differences between the performance of each of the EM and MM groups in Q1 and Q2, Q1 and Q3, and Q2 and Q3.

The quantitative analysis was supplemented by the qualitative analysis of the data gathered from the interview transcripts and the written explanations given in the test. The classifications of responses were guided by students' choices in the multiple-choice questions. The selection of student responses to be presented in the following section was guided by the following two criteria: (a) illumination of students' thinking, and (b) illustration of the variety of students' reasoning strategies.

\section{Results}

The results are organized in three parts, each corresponding to one research question. Even though the three research questions are closely related, we keep this structure in presenting the results for the sake of clarity. In the last section of the paper, the research questions are connected and discussed integrally.

First, we report the percentages of students' responses in each of the multiple-choice questions, and we use students' written and oral explanations to further investigate their understanding of contraposition. To reduce the likelihood of improper generalizations, percentages of types of student explanations that exhibit particular characteristics are not reported. In the other two parts, we present the results of statistical analysis on students' performance in the three tasks.

\subsection{Students' understanding of contraposition}

The percentages within major for each of the response options in Q1 are presented in Table II. A general comment, which will be further investigated in the next part, is that MM did considerably better than EM in Q1. Almost two thirds (64.0\%) of the MM gave a correct response to Q1, as compared to only one fifth of the EM. Approximately half $(47.1 \%)$ of the EM considered the proposed proof by contraposition as false, whereas only $28.0 \%$ of the MM expressed the same opinion. The rest of the EM were divided almost equally among the options "The proof shows that 
TABLE II

Percentages for each response option in question 1 by major

\begin{tabular}{lcc}
\hline Response in Question 1 & \multicolumn{2}{l}{ Major } \\
\cline { 2 - 3 } & Education $^{\mathrm{a}}$ & Mathematics $^{\mathrm{b}}$ \\
\hline The proof is false & 47.1 & 28.0 \\
The proof shows that the statement is always true & 20.0 & 64.0 \\
The proof shows that the statement is true in some cases & 15.7 & 4.0 \\
I have no opinion & 17.1 & 4.0 \\
\hline Total & 100.0 & 100.0 \\
\hline
\end{tabular}

Note. The values represent percentages within major.

${ }^{\mathrm{a}} \mathrm{n}=70 .{ }^{\mathrm{b}} \mathrm{n}=25 .{ }^{\mathrm{c}}$ Correct response for question 1 .

the statement is true in some cases" and "I have no opinion" (15.7\% and $17.1 \%$, respectively). Each of these two options was selected by only one MM (this corresponds to $4.0 \%$ of the MM participants).

From the explanations that EM gave in the interviews, it is clear that one of the reasons that made them consider the contraposition proof as wrong was that it was short. Below is an extract from the interview with EM38 ('I' denotes the interviewers).

I: $\quad$ In the first question, you stated that the proposed proof was wrong. Could you please explain your reasoning?

EM38: I said that it was wrong because of its appearance. This, at least, doesn't inspire me to say that the proof is correct. I believe that it is too short to be a proof.

A number of EM rejected the proposed proof by disputing its generality over the whole set of natural numbers.

EM36: I believe that the calculations in the proof are correct. However, the proof does not convince us that the statement is valid in the general case where $x$ and $y$ are natural numbers.

I: $\quad$ So, do you think that the proof is a right or a wrong one for the given statement?

EM36: In my view... I think that it is not a correct proof.

Some students did not notice that $x, y \in \mathbf{N}$ and assumed that $x, y \in$ $\mathbf{Z}$; this often made them choose the option "The proof shows that the statement is true in some cases", as they believed that the statement failed for pairs of opposite integers. However, even if $x$ and $y$ belonged to the set of integers, the proof would still hold. 
EM18: The way the statement [in the proof] is presented, it is valid for some numbers... for some values of $x$ and $y$ it is true and for some other values it is not true. For example, we know that two numbers... which have equal square values... these numbers are equal. However, it is possible for two numbers to have the same absolute value, hence their square values are equal, but these numbers are not equal. For example, $x$ could be equal to -5 and $y$ could be equal to +5 .

MM12: The proof is wrong because, even though there is no error in it, it doesn't show the required. In other words, the proof is correct, but it doesn't prove what it was supposed to prove.

I: $\quad$ Which case does the proof fail to examine?

MM12: The case of the negation. For example, when we have a number that is negative with its opposite number. For instance, take -3 with +3 .

Students' confusion seems to originate from their failure to realize that neither the statement nor its proof make use of the implication $x^{2}=y^{2} \Rightarrow$ $x=y$, which indeed requires the condition $x, y \in \mathbf{N}$ in order to be true (given that we can only choose between the sets of natural numbers and integers). This confusion may be associated with the belief that the correct logical equivalence is $p \Rightarrow q \equiv \sim p \Rightarrow \sim q$ and not that of contraposition. It is also worth noting that both EM18 and MM12 seem to readily accept the statement that 'If $x=y \Rightarrow x^{2}=y^{2}$, which was given in the proposed proof; this provides further support to the claim that the reason for which these students did not accept that the proof was correct was that they did not believe that the given implication statement and its contrapositive were equivalent.

During the interviews, we pointed out to the students the given they were 'looking for' (i.e., that $x, y \in \mathbf{N}$ ). This information caused some of them to revise their initial response (e.g., EM18); this, however, does not necessarily imply that they also understood the logic of the proof.

I: $\quad$ If you look back at the statement, however, both $x$ and $y$ are natural numbers. As a result, they can only be positive.

EM18: Oh...they are both positive. Yes, I think that, given that both $x$ and $y$ are positive, the statement is complete. In other words, the statement always holds. It always holds, because it is not possible now to have two equal numbers with different square values.

I: $\quad$ Do you think that the proposed proof proves the statement? 
EM18: The given proof should be part of a more general proof that would include 'analysis' and 'synthesis'. What I mean is that here we proceed backwards. We conduct an examination and say that $x=y$ implies... $x^{2}=y^{2}$. The proof should then use this and say that, if $x^{2} \neq y^{2}$ then $x \neq y$, since both $x$ and $y$ are natural numbers.

EM18 seems to believe that the proof under examination should be part of a more detailed proof, as the additional step he suggests is, basically, a remark that the required has been shown.

Contrary to what happened with EM18, MM12 insisted on his original view that the proof is false even after we pointed out to him the unnoticed given.

I: $\quad$.. Yes, but the statement says that both $x$ and $y$ are natural numbers.

MM12: I probably didn't notice that.

I: $\quad$ Does this change anything to you?

MM12: I continue to believe that the proof is wrong, because I think that it proves the other direction, the converse of the required. Because... it proves that if $x \neq y$ then $x^{2} \neq \mathrm{y}^{2}$. Basically, using the method of contradiction it proves the converse. I continue to believe that it doesn't prove what it was supposed to prove.

The above extract suggests that MM12 does not accept the validity of the contraposition equivalence rule. The student claims that the suggested proof shows that 'if $x \neq y$ then $x^{2} \neq y^{2}$, and not the required, that is, 'if $x^{2} \neq y^{2}$ then $x \neq y^{\prime}$. Given the connection that the student makes between the statement used in the proof (i.e., 'if $x=y$ then $x^{2}=y^{2}$ ') and the proposition 'if $x \neq y$ then $x^{2} \neq y^{2}$, he seems to think that the former is equivalent to the latter. This provides further support to the claim that the student believes that the following 'logical equivalence' is true: $p \Rightarrow q \equiv \sim p \Rightarrow \sim q$. Finally, the student's response also reveals confusion about proof by contradiction.

Table III shows the percentages per major for each of the response options in Questions 2 and 3. A general comment from the table is that the MM did better than the EM in Q2, while the reverse happened in Q3 (these observations are investigated statistically in the following part). As regards the EM, 67.1\% answered correctly that the conclusion in Q2 was correct, $22.9 \%$ considered it incorrect, and the rest $(10.0 \%)$ replied that they did not have enough information to decide. The corresponding percentages for the $\mathrm{MM}$ in this question were $76.0 \%, 20.0 \%$, and $4.0 \%$. In Q3, $75.7 \%$ of the EM considered correctly the conclusion as false, $15.7 \%$ regarded it as true, and the remaining $8.6 \%$ said that they could not decide from the given clues. The percentages for the MM were $60.0 \%, 4.0 \%$, and $36.0 \%$, respectively. 
TABLE III

Percentages for each response option in questions 2 and 3 by major

\begin{tabular}{lccccc}
\hline \multirow{2}{*}{ Responses } & Question 2 & & \multicolumn{2}{l}{ Question 3 } & \\
\cline { 2 - 3 } \cline { 5 - 5 } & Education $^{\mathrm{a}}$ & Mathematics $^{\mathrm{b}}$ & & Education $^{\mathrm{a}}$ & Mathematics $^{\mathrm{b}}$ \\
\hline The conclusion is correct $^{\mathrm{c}}$ & 67.1 & 76.0 & 15.7 & 4.0 \\
The conclusion is wrong $^{\mathrm{d}}$ & 22.9 & 20.0 & 75.7 & 60.0 \\
$\begin{array}{l}\text { I do not have enough clues } \\
\text { to decide }\end{array}$ & 10.0 & 4.0 & 8.6 & 36.0 \\
\hline Total & 100.0 & 100.0 & 100.0 & 100.0
\end{tabular}

Note. The values represent percentages within major.

${ }^{a} n=70 .{ }^{b} n=25 .{ }^{c}$ Correct response for question $2 .{ }^{d}$ Correct response for question 3.

The students that considered the conclusion of Q2 as correct justified their decisions in many ways. Some of these justifications were clearly making use of the contraposition equivalence rule (e.g., EM12).

EM12: $\quad \mathrm{A}($ pneumonia $) \Rightarrow \mathrm{B}$ (high fever) $\equiv$ not $\mathrm{B} \Rightarrow$ not $\mathrm{A}$.

Some other responses could be considered more or less as repetitions of the givens and the conclusion (e.g., EM60, EM24); from this kind of responses it is hard to say whether the students really believed in the validity of the contraposition equivalence rule.

EM60: It [i.e., the conclusion in $\mathrm{Q} 2]$ is correct. If he suffers from pneumonia, then he certainly has fever. If he doesn't have fever, then he certainly doesn't suffer from pneumonia.

EM24: High fever is a definite symptom of pneumonia. If he has no fever, the disease cannot be pneumonia.

Other students used logical thinking and concepts of set theory (Venn Diagrams) to justify their responses (e.g., EM49, MM2); these justifications are more revealing of the students' way of thinking.

EM49: $\quad$ Since high fever (A) is a certain consequence of pneumonia (B), then B is a subset of A. Hence, if A doesn't hold, B doesn't hold either. Therefore, the conclusion is correct.

MM2: $\quad$ It is correct.



A: Costas has pneumonia

B: Costas has high fever 
Finally, MM1 used reasoning by contradiction to justify the validity of the given conclusion. This explanation illustrates the relation between proof by contradiction and contraposition equivalence rule.

MM1: It is true. It's an application of proof by contradiction. If he suffered from pneumonia, he would have high fever. Contradiction, since he doesn't have high fever.

Some students argued that the conclusion was incorrect (e.g., EM2, EM58, MM7) or that they did not have enough clues to decide (e.g., EM8), because they did not accept the equivalence of the two conditional sentences. It is worth noting that one of the students (EM2) who considered the conclusion incorrect referred explicitly to contraposition.

EM2: 'Costas suffers from pneumonia' is a statement that implies the statement 'He has high fever'. The conclusion, however, is wrong because this doesn't necessarily mean that the contrapositive holds too.

EM58: It is wrong. We know that 'If a, then b'. Now we are given 'not b'. This does not imply 'not a'.

MM7: $\quad$ Pneumonia $\Rightarrow$ High fever. Doesn’t suffer from pneumonia $\neq \mathrm{He}$ doesn't have high fever.

EM8: I cannot decide. It does not necessarily mean that if B doesn't hold, then A doesn't hold either.

Some students (e.g., EM13, EM50) considered the conclusion incorrect, because it was not compatible with their real-life knowledge and experience about pneumonia and fever. These students did not manage to decontextualize the statements and assess the necessity of the conclusion in purely logical terms.

EM13: Perhaps fever is not the only symptom. Therefore, the conclusion is wrong.

EM50: The conclusion is false. Perhaps Costas 'lost' his voice because of pneumonia or inflammation. Fever is not the only symptom of pneumonia.

However, the reasoning behind these statements is incorrect; the conclusion would hold even in the case that high fever were not the only symptom of pneumonia.

In the interviews, we tried to explore whether the students could see the connection between Q1 and Q2. In the cases where the subjects accepted the validity of the contraposition equivalence rule only in the verbal context of Q2, we tried to see whether the discussion would help them also accept its validity in the symbolic context of Q1. The following extract from the interview with EM36 is such an example. 
I: $\quad$ Can you go back now and read again question 2, in order to see whether there is any resemblance between that problem and the proof we have just talked about in question 1 ?

EM36: If we assign mathematical symbols to the two statements [of Q2], then they [i.e., Q1 and Q2] look similar. Even though in question 2 I gave a correct response and accepted... the solution as true, in question 1 I didn't make something similar.

I: $\quad$ Why do you think that happened?

EM36: Because it's a more abstract concept and I didn't have any concrete point of reference.

EM36 attributes his inconsistency in the way he responded to the two questions to the concreteness of the verbal context as opposed to the abstractness of the symbolic notation. Along similar lines EM38 talks about the 'clarity' of the verbal context:

EM38: Perhaps here [i.e., Q2] I wrote this [i.e., the correct answer] because of the clearer character of the task. In my view, the verbal context is clearer.

I: $\quad$ Are you saying that you can see a resemblance between these statements [i.e., the statements in Q2] and the proof in question 1 ?

EM38: Yes. We have again the contrapositive. One assumes the negation of the second and concludes with the negation of the first. I believe that the proof in question 1 is also correct.

It is worth noting that both EM36 and EM38, after noticing the inconsistency in their responses to Q1 and Q2, supposed that the correct choice was the one given to the question placed in the verbal context (namely, Q2) and started looking for reasons that caused their incorrect response to the symbolic task (namely, Q1).

Several students correctly revised their thinking about the validity of the proof in Q1 after the cognitive conflict they experienced with the realization that they responded differently to two isomorphic problems. Of course, there were also some students who had difficulty understanding the same logical structure of the verbal and the symbolic tasks. For instance, MM12 was unwilling to accept the correctness of the proof in Q1, even after he had the chance to reconsider, during the interview, his original response to Q2 and seemed to have accepted the correctness of the conclusion. 
MM12: Looking again at the conclusion [of Q2], I now believe that it is true because high fever is necessary condition for Costas to have pneumonia.

I: $\quad$ Can you see any resemblance between this conclusion and the proof in question 1 ?

MM12: I believe that the correct response would be to say that ' $x \Rightarrow y$ ' is equivalent to 'not $x \Rightarrow$ not $y$ ' and not that it implies the other, i.e., that 'not $y \Rightarrow \operatorname{not} x$ '.

Our discussion with MM19 was along the same lines as that with MM12. MM19 accepted originally the validity of the conclusion in Q2, but rejected the proof in Q1 because of his belief that the contraposition equivalence rule was wrong. Contrary to what happened with MM12, though, MM19 used this belief to (implicitly) revise his response to Q2 saying that the conclusion "Costas definitely does not suffer from pneumonia" does not follow from the two statements.

MM19: The proof in question 1 says that, 'if $x=y$ then $x^{2}=y^{2}$. It doesn't say that, 'if $x \neq y$ then $x^{2} \neq y^{2}$, We only have implication here and not equivalence. This proof is incomplete. It's wrong.

I: $\quad$ Okay. In question 2 you said that the conclusion is correct. Suppose we see now question 2 in terms of 'implications' [expressed in symbolic notation]. Can you see any resemblance between question 2 and the proof in question 1 we discussed earlier?

MM19: If we set $p$ to mean 'Costas suffers from pneumonia' and $q$ to mean 'Costas has high fever', the statement means that 'if $p$ then $q$ '. 'Costas doesn't have high fever' is the negation of $q$. The negation of $q$ doesn't imply the negation of $p$.

The extracts from the interviews with both MM12 and MM19 reveal that some students reject the contraposition equivalence rule because they believe that the correct equivalence relating the conditional sentence $p \Rightarrow q$ with the propositions $\sim p$ and $\sim q$ is $p \Rightarrow q \equiv \sim p \Rightarrow \sim q$. For more integrated investigation of students' understanding, we next examine students' responses to $\mathrm{Q} 3$.

Below, we present some examples of written responses from students who stated that the conclusion of Q3 was wrong.

E61: $\quad$ It is wrong: $\sim p \Rightarrow \sim q \equiv p \Rightarrow q$.

MM3: $\quad$ It is incorrect. 


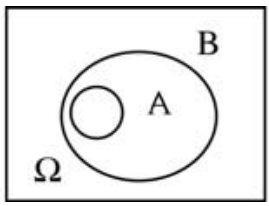

A: The car can move

B: The car has fuel

EM20: It is false. The statement says that without fuel the car won't move, and not that when the car has fuel (and only then) will it definitely move.

EM24: It is false. The statements don't imply that the only condition for the car to move is to have fuel.

EM55: It is false. Fuel is a basic (perhaps the most important) condition for a car to move. It is not the only one, however. Therefore, it doesn't necessarily follow that the car can move just because it has fuel. For instance, the car may have a mechanical problem.

It is plausible to say that EM55 rejects the conclusion on the basis of everyday life experiences and not on logical rules of inference.

Several students who chose the option "I do not have enough clues to decide" justified their responses by arguing that they could not make a definite decision about the necessity of the conclusion in $p \Rightarrow q$ given that both $\sim p \Rightarrow \sim q$ and $p$ hold. Several of them failed to recognize that this conclusion was phrased (in Q3) as a logically necessary inference and was, therefore, wrong.

EM58: I cannot decide, because the statement 'if not $a \Rightarrow$ not $b$ ' does not give us any information about the 'validity of $a$ '.

MM1: I cannot decide because we are not told what follows from the fact that 'the car has fuel'.

MM5: The first [statement in Q3] is an implication and not an equivalence, hence I do not have enough clues to decide.

The students who accepted the validity of the conclusion explained their reasoning by relying basically on the wrong equivalence $\sim p \Rightarrow \sim q \equiv p \Rightarrow q$.

E35: Without fuel, the car definitely cannot move. Hence, since it has fuel, the conclusion is correct.

Others seemed to know what the correct logical operation should be, but failed to realize that translating Q3 into symbolic notation would actually yield a different and, at the same time, wrong logical equivalence. The response of E12 is indicative.

$$
\text { E12: It is true: Not } \mathrm{A} \rightarrow \text { Not } \mathrm{B} \equiv \mathrm{B} \rightarrow \mathrm{A}
$$

As with the other questions, there were some students who based their responses on their everyday experience (e.g., EM14, EM46, MM7). 


\section{TABLE IV}

Percentages of correct and incorrect responses to questions 1, 2, and 3 by major, and for the total sample

\begin{tabular}{|c|c|c|c|c|c|c|}
\hline \multirow[t]{3}{*}{ Major } & \multicolumn{2}{|l|}{ Question 1} & \multicolumn{2}{|l|}{ Question 2} & \multicolumn{2}{|l|}{ Question 3} \\
\hline & False & Correct & False & Correct & False & Correct \\
\hline & Response & Response & Response & Response & Response & Response \\
\hline Education $^{\mathrm{a}}$ & 80.0 & 20.0 & 32.9 & 67.1 & 24.3 & 75.7 \\
\hline Mathematics $^{\mathrm{b}}$ & 36.0 & 64.0 & 24.0 & 76.0 & 40.0 & 60.0 \\
\hline Total Sample ${ }^{c}$ & 68.4 & 31.6 & 30.5 & 69.5 & 28.4 & 71.6 \\
\hline
\end{tabular}

Note. The values represent percentages within major in each question.

${ }^{\mathrm{a}} \mathrm{n}=70 .{ }^{\mathrm{b}} \mathrm{n}=25 .^{\mathrm{c}} \mathrm{n}=95$.

\section{TABLE V}

Comparisons of percentages of correct responses for the total sample

\begin{tabular}{llll}
\hline & \multicolumn{3}{l}{ Question } \\
\cline { 2 - 4 } Question & 1 & 2 & 3 \\
\hline 1 & - & $-6.30^{*}$ & $-6.07^{*}$ \\
2 & & - & -.35 \\
3 & & & - \\
\hline
\end{tabular}

Note. The values represent $t$-values in the two-tailed paired samples tests for differences in percentages of correct responses in every combination of 2 for the three questions. $\mathrm{n}=95$, df $=94 .{ }^{*} p<.001$. The unmarked value was not found to be statistically significant $(p>.05)$.

E14: The car will move, but this is not for sure. Even though it has fuel, there still may be a mechanical problem. The conclusion is only partly true.

E46: It is true, but not definitely [true]. Something may happen and the car may not move (for other reasons [not related to fuel]).

MM7: It is true given that there are no other problems or factors affecting the movement of the car.

\subsection{Relation between students' performance and context}

Table IV summarizes the percentages of correct and incorrect responses to Questions 1, 2, and 3 by major as well as for the total sample. Table V 
TABLE VI

Comparisons of percentages of correct responses for the two majors

\begin{tabular}{llllllll}
\hline & \multicolumn{3}{c}{ Education $^{\mathrm{a}}$} & & \multicolumn{3}{c}{ Mathematics $^{\mathrm{b}}$} \\
\cline { 2 - 4 } \cline { 4 - 7 } Question & 1 & 2 & 3 & & 2 & 3 \\
\hline 1 & - & $-6.77^{*}$ & $-7.70^{*}$ & - & -1.14 & .37 \\
2 & & - & -1.29 & & - & 1.28 \\
3 & & & - & & & - \\
\hline
\end{tabular}

Note. The values represent $t$-values in the two-tailed paired samples tests for differences in percentages of correct responses in every combination of 2 for the three questions.

${ }^{\mathrm{a}} \mathrm{n}=70, \mathrm{df}=69 .{ }^{\mathrm{b}} \mathrm{n}=25, \mathrm{df}=24 .{ }^{*} p<.001$. The unmarked values were not found to be statistically significant $(p>.05)$.

presents the results of $t$-tests that were carried out to investigate the difference between the performances in every combination of two questions for the group of 95 participants as a whole. As is shown in this table, the percentage of students who answered correctly Q1 was significantly different from the corresponding percentages for both Q2 and Q3 $(t(94)=-$ $6.30, p<.001$, and $t(94)=-6.07, p<.001$, respectively). Taking into account the actual percentages of correct responses to the three questions $(31.6 \%$, 69.5\%, and 71.6\% for Q1, Q2, and Q3, respectively; see Table IV), we can conclude that students performed significantly better in the verbal context (Q2 and Q3) than in the symbolic (Q1). There is no evidence to suggest that students' performance was different in the two verbal questions, Q2 and Q3 $(t(94)=-.35, p=.73)$.

\subsection{Relation between students' major and performance in different contexts}

Chi-Square test of Independence showed that there is a relation between performance in Q1 (PQ1) and the students' major (Pearson $\chi^{2}(1, \mathrm{~N}=95)=$ $16.51, p<.001)$. Looking at how the percentages were distributed (Table IV), we may conclude that MM are more likely than EM to do better in Q1. Chi-Square tests of Independence revealed no significant relation between PQ2 and PQ3, and students' major (Pearson $\chi^{2}(1, N=95)=.68$, $p=.41$, and Pearson $\chi^{2}(1, \mathrm{~N}=95)=2.24, p=.14$, respectively $)$.

Table VI summarizes the results of $t$-tests that were carried out to investigate the difference between the performances in every combination of two questions for each of the two groups (EM and MM) separately. As is shown in the table, the percentage of EM who answered correctly Q1 was significantly different from the corresponding percentages of both 
Q2 and Q3 ( $t(69)=-6.77, p<.001$ and $t(69)=-7.70, p<.001$, respectively). Taking into account the actual percentages of correct responses in the three questions $(20.0 \%, 67.1 \%$, and $75.7 \%$ for Q1, Q2, and Q3, respectively; see Table IV), we can conclude that EM performed significantly better in the verbal context (Q2 and Q3) than in the symbolic (Q1). There is no evidence to suggest that the performance of EM was different in the two verbal questions, Q2 and Q3 $(t(69)=-1.29, p=.20)$. There is also no evidence to suggest that MM performed differently in any of the combinations of two questions in the test: Q1-Q2 $(t(24)=-1.14, p=.27), \mathrm{Q} 1-\mathrm{Q} 3(t(24)=.37$, $p=.71), \mathrm{Q} 2-\mathrm{Q} 3(t(24)=1.28, p=.21)$.

\section{Discussion AND CONCLUSIONS}

The results suggest that students' major (education/mathematics) does play a role in students' performance in contraposition placed in different contexts (symbolic/verbal). When the participants were considered as a whole, there was evidence that their performance in contraposition was significantly better in the verbal than in the symbolic context $(31.6 \%$ and $69.5 \%$, respectively). Their performance in the two verbal tasks about contraposition was comparably equal $(69.5 \%$ and $71.6 \%)$. These results could be taken as evidence in favor of the claim that logical competence in the symbolic reasoning domain is, in general, lower compared to that in other reasoning domains, such as verbal (Morris, 2002). When the participants were considered according to their majors, however, the picture was not the same for both EM and MM. Only the performance of the EM was in complete agreement with the general picture reported earlier; the performance of the MM deviated from the above pattern as it presented only modest variations between the two contexts. The latter remark suggests that MM operate comparably the same in symbolic and verbal reasoning domains related to contraposition. Furthermore, the fact that the two groups' performance showed different variations in the two contexts, points out a merit for considering the two student populations separately. The finding that the general picture of students' performance agrees with that of the education majors, when the two groups are considered separately, can primarily be attributed to the considerably larger representation of EM in the sample compared to the representation of MM (this, despite the fact that the MM that participated in the study constituted the whole population of the seniors of the Mathematics Department of the University of Cyprus that specific academic year).

The analysis of the data showed that EM performed significantly better in the verbal contexts of Q2 and Q3 - which examined, respectively, the 
equivalence (or not) of a logical implication, on the one hand, and its contrapositive or inverse, on the other - compared to their performance in the symbolic context of Q1 - which examined the contraposition equivalence rule in the context of a mathematical proof. In particular, the EM performed comparably equal in the two verbal tasks $(67.1 \%$ in Q2 and $75.7 \%$ in Q3) and more than three times better than in the symbolic task $(20.0 \%$ in Q1). It is worth noting that one of the reasons that the EM did not accept the validity of the contraposition proof in Q1 was that the suggested proof was 'short'. This proof characteristic made several EM consider the proof as non-convincing and regard its argument as being superficial. The results support the findings of other studies with students majoring in education. Martin and Harel (1989) found that the judgments of a mathematical argument by many pre-service elementary school teachers were influenced by its appearance in the proof context - what they called the ritualistic aspects of proof - and not the correctness of the argument. Along similar lines, Winicki-Landman (1998) reports that students majoring in education were found to employ aesthetic criteria, such as length and explanatory power, in evaluating the validity of a proof. We need to note, however, that reliance on ritualistic aspects of a proof in judging its validity is not a tendency exclusively associated with the mathematical behavior of education majors; similar results are reported for other student populations as well, such as high-school students (Vinner, 1983) and mathematics majors (Harel and Sowder, 1998). Harel and Sowder (1998), for example, describe an episode in which a student in an elementary Linear Algebra course, taken by sophomore mathematics majors, had doubts whether one of his proofs was mathematically valid, because, as he noted, it did not 'look like' a proof. Furthermore, there is evidence that students employ similar criteria while making mathematical decisions in other domains as well, such as the domain of functions, graphs, and graphing. In particular, research has shown that students tend to connect points (regardless of the appropriateness of this action) because it 'looks better' (Leinhardt et al., 1990).

The analysis of the data collected from the MM students showed that, compared to their performance in the task placed in symbolic context (Q1), they did better in the verbal context of Q2 and slightly worse in that of Q3; the percentages, however, were close enough to each other $(64.0 \%, 76.0 \%$, and $60.0 \%$, respectively) so that no statistically significant differences were found. A plausible interpretation would be that MM appear to be equally competent in reasoning about contraposition in symbolic and verbal contexts. Even though the results provide some support to this interpretation, the small number of MM that participated in this 
study do not allow for a thorough investigation of this issue. The qualitative analysis of our data revealed that one of the reasons that led MM to reject the contraposition proof was the belief that the correct logical equivalence was $p \Rightarrow q \equiv \sim p \Rightarrow \sim q$ and not that of contraposition. This finding is consistent with Knuth's (2002) findings from a study with 16 secondary school mathematics teachers all of whom had undergraduate degrees in mathematics or in related fields. Specifically, ten of the participants in Knuth's study accepted as proof a mathematical argument that was based on the same invalid logical equivalence. In future investigations, larger numbers of participants majoring in mathematics are necessary to address the methodological constraints imposed by the small sample sizes in both our and Knuth's study. We also suggest that future studies of this kind include both symbolic and verbal tasks for the invalid logical equivalence $p \Rightarrow q \equiv \sim p \Rightarrow \sim q$. Had we asked the participants to also react to the 'proof' given below for the statement of Q1, we would be in a better position to investigate students' understanding of contraposition, and the role of the context (wherein logical equivalences are placed) in students' performance:

Statement: If $x^{2} \neq y^{2}$ then $x \neq y$ (where $x, y \in \mathbf{N}$ )

Proof: If $x^{2}=y^{2} \Rightarrow x=y$. Hence, the statement is true.

A number of students judged the validity of the conclusions in the verbal tasks (Q2 and Q3) not by considering whether the given statements logically necessitate the conclusions, but by introducing personal knowledge about the relations between pneumonia and high fever, in one of the tasks (Q2), and fuel and car movement, in the other (Q3). This finding is compatible with earlier observations that adult reasoners' application of knowledge about judgments of necessity is affected by personal knowledge or beliefs about the argument content (Morris, 2002). The meaningful ${ }^{6}$ words and relations used in the verbal tasks allowed students to draw from their experiences and personal knowledge in judging the validity of the suggested statement-conclusion relations; the prior knowledge and experiences sometimes proved to be helpful and others misleading. An interesting investigation would be to also give students statements with non-meaningful words and see how this affects their performance. The results of previous research (O'Brien, 1972) suggest that, in the case of tasks with non-meaningful words, students' performance is likely to decrease. Research, however, provides a weak basis on which to formulate hypotheses about the relation between students' performance in tasks with non-meaningful words and symbolic tasks that investigate the same logical principles. Also, research provides little information about possible differences in the performances of students majoring in different fields of 
study on such tasks. Although students' performance in tasks with nonmeaningful words has a research interest (in terms of exploring further students' logical competence), the examination of their performance in meaningful verbal contexts appears to be pedagogically more important (Epp, 1998).

The fact that MM performed significantly better than EM in the symbolic task is not surprising. The frequency with which contraposition equivalence rule is encountered in the mathematics program of study is quite high; contraposition equivalence rule is frequently used in proving universal conditionals of the form $\forall x \in D$, if $P(x)$ then $Q(x)$, and the rationale that lies behind it also relates to those of other proof methods repeatedly encountered in the mathematics collegiate curriculum, namely, proof by contradiction and proof by counterexample. Although EM performed poorly in the symbolic task (Q1), they performed comparably equal with $\mathrm{MM}$ in the questions placed in verbal context $(\mathrm{Q} 2$ and Q3) and they even outperformed $\mathrm{MM}$ in one of them (Q3). It is plausible to argue that this dramatic increase in the performance of the EM in the verbal tasks reflects the emphasis of the education program of study, wherein, even though symbolic manipulations are not ignored, the focus is more on verbal, reallife problems. Problems that involve use of symbolic notation, primarily found in the probability and the two foundations courses of the mathematics curriculum of the Department of Education (cf. section 2.1), are often connected to verbal situations in an attempt to make them more relevant to the work of teaching elementary mathematics. The last in the sequence of four courses relevant to mathematics taken by EM, namely, the course on the teaching and learning of mathematics, focuses on problems that require more verbal syllogistic reasoning and fewer justifications that are strictly based on mathematical symbolism. The relatively uniform performance of the MM in the symbolic and verbal problems is harder to explain with reference to the presumed emphasis of the mathematics collegiate curriculum on symbolic tasks. One plausible explanation might be that MM were able to apply their symbolic knowledge of contraposition in the verbal tasks as well; this explanation is grounded on the assumption that MM intentionally tried and were successful in transferring their symbolic knowledge to the verbal tasks. This interpretation is supported by the results that showed that the reasons for which MM rejected Q2 were very similar to those for which they rejected Q1; these reasons were centered on the belief that an implication statement is equivalent to its inverse rather than to its contrapositive. The validity of this interpretation is, however, questioned if one considers the interview with MM12 that revealed his difficulties to relate problems 
Q1 and Q2. These issues can only be addressed with future research that will include more interviews with MM participants.

The results of this study call for both major- and context- specific considerations of students' understanding of, and performance in, logical principles. The students' major and the context wherein a task is placed are not the only parameters that appear important to consider when reporting on students' reasoning on logical principles. For instance, students' mathematical competence and views of proof were also found to relate to their understanding of proof (Healy and Hoyles, 2000). On the other hand, there is evidence that the mathematical content (arithmetical/geometrical) of the problems has little influence on students' mathematical proof capacity (Recio and Godino, 2001). We need, however, to be cautious when drawing conclusions about the relevant importance of factors that have been examined in different studies, given the diversity in populations and methodological practices. It is often the case that factors examined in some studies are not considered in others. Take, for example, the study by Recio and Godino (2001). In this study, one of the samples consisted of 429 freshmen that took a mathematics subject in different faculties and polytechnic schools. Even though students' proof schemes appeared to be relatively independent from the mathematical content (arithmetical/geometrical) of the problems posed, the findings of the present study suggest that the same picture might have not been obtained had the students been considered according to their majors. Because Recio and Godino's sample consisted of freshmen at the beginning of their studies, students' major would presumably reflect the emphases of the high-school mathematics curricula that prepared students for entering the different faculties and polytechnic schools, rather than the emphases of the collegiate curricula of these faculties and schools. Similar reasoning to the above could be applied to suggest that, because in our study the mathematical proof presented in Q1 had an arithmetical content (in Recio and Godino's terms), it is uncertain whether the same results would be obtained had the content been geometrical.

Furthermore, it is important to recognize that the mathematical preparation received by the students that participated in our study may differ from the one received by students majoring in the same fields but in other countries; this may be particularly true about the mathematical preparation of education majors. This remark introduces another factor, namely, the cultural environment in which the study is conducted; this factor might be important to consider, for it has been found to play a role in other domains, such as the one of solving arithmetic problems. Specifically, Brazilian students were found to be more successful when solving arithmetic problems 
in word context than when solving equivalent but purely symbolic problems (Carraher et al., 1987). However, a study with U.S. children (Baranes et al., 1989) that used the same experimental paradigm of the Brazilian study by Carraher et al. (1987) did not replicate these results - "no effects of context were found in either strategy use or success" (Baranes et al., 1989, p. 287). The context was found to be important only when additional factors were also considered; for example, the numbers were found to "interact with problem content in a way that may or may not facilitate successful solution of the problem" (Baranes et al., 1989, p. 316).

The apparent complexity of the system of factors that influence students' understanding of logical principles gives rise to the following question: To what extent should we consider different factors in examining students' conceptualizations of the structure of logical relations? We claim that the level of specificity should depend on the extent to which distinctive and meaningful patterns are obtained. This study suggests that students' major as well as the context (verbal/symbolic) wherein a logical task is placed are two factors to consider in conducting such investigations.

Besides the research implications, our study also suggests a useful pedagogical implication. The teaching of logical principles may vary with student populations, especially when students are well advanced in their program of studies. If, for example, students' ability to reason about logical principles is, or is assumed to be, higher in verbal than in symbolic contexts (as it was the case with the EM in our study), then it is plausible to argue that instruction should build on students' strengths in the verbal reasoning domain to help them develop their capacity to reason in structurally similar symbolic domains.

\section{ACKNOWLEDGEMENTS}

This paper is based on the senior thesis of the first two authors conducted at the University of Cyprus, under the supervision of the third author; the order of authorship of the first two authors is alphabetical. The authors wish to thank, gratefully, Deborah Ball and Hyman Bass for their helpful comments on an earlier version of this paper.

\section{NOTES}

1. When we talk about contraposition equivalence rule in symbolic context, we basically refer to proof by contraposition.

2. Although there is relatively little mathematics education research in the area of formal and pragmatic logic, there is a considerable number of studies in other fields, especially in psychology, that concentrate on this area (see, e.g., Cheng et al., 1986; Girotto et al., 1988; Johnson-Laird et al., 1972). Because of the different focuses of mathematics education research and other kinds of research, we consider important to have more 
studies that look specifically to issues of teaching and learning of mathematics in the area of formal and pragmatic logic.

3. Papanastasiou and Papanastasiou (1997) also compare the factors that have influenced students at the Pennsylvania State University and at the University of Cyprus to major in elementary education.

4. These courses cover material that include the number systems and their main characteristics, Pythagorean theorem, Ptolemy's theorem and the birth of trigonometry, Pappus's theorem, Fibonacci sequence, the solution of third- and fourth-degree equations, the development of differential and integral calculus, elements of non-Euclidean geometries, acclimatization to algebra, elements of matrix algebra, Boolean algebra, number theory, Cartesian products, binary relations, functions, logical propositions, algebra propositions, logic gates, and logic circuits. For a more detailed description of the two courses see Philippou and Christou (1998) or the following web page: http://www.ucy.ac.cy/epa/coursesE/sthirdareaE/sthirdareae.html.

5. The original items were in Greek. The same holds for the students' responses to be presented in the next section.

6. In our sense, meaningful does not necessarily imply realistic.

\section{REFERENCES}

Ball, D.L. and Bass, H.: 2000, 'Making believe: The collective construction of public mathematical knowledge in the elementary classroom', in D. Philips (ed.), Constructivism in Education: Yearbook of the National Society for the Study of Education, University of Chicago Press, Chicago, pp. 193-224.

Ball, D.L. and Bass, H.: 2003, 'Making mathematics reasonable in school', in J. Kilpatrick, W.G. Martin and D. Schifter (eds.), A Research Companion to Principles and Standards for School Mathematics, National Council of Teachers of Mathematics, Reston, VA, pp. 27-44.

Baranes, R., Perry, M. and Stigler, J.: 1989, 'Activation of real-world knowledge in the solution of word problems', Cognition and Instruction 6(4), 287-318.

Braine, M.D.S. and Rumain, R.: 1983, 'Logical reasoning', in J.H. Flavell and E.M. Markman (eds.), Carmichael's Handbook of Child Psychology: Vol. III. Cognitive Development, Wiley, New York, pp. 263-340.

Carpenter, T.P., Franke, M.L. and Levi, L.: 2003, Thinking Mathematically: Integrating Arithmetic and Algebra in Elementary School, Heinemann, Portsmouth, NH.

Carraher, T.N., Carraher, D.W. and Schliemann, A.D.: 1987, 'Written and oral mathematics', Journal for Research in Mathematics Education 18, 83-97.

Chazan, D.: 1993, 'High school geometry students' justification for their views of empirical evidence and mathematical proof', Educational Studies in Mathematics 24, 359-387.

Cheng, P.W., Holyoak, K.J., Nisbett, R.E. and Oliver, L.M.: 1986, 'Pragmatic versus syntactic approaches to training deductive reasoning', Cognitive Psychology 18, 293-328.

Coe, R. and Ruthven, K.: 1994, 'Proof practices and constructs of advanced mathematics students', British Educational Research Journal 24(4), 333-344.

Dubinsky, E.: 1986, 'Teaching mathematical induction I', Journal of Mathematical Behavior 5, 305-317.

Dubinsky, E.: 1990, 'Teaching mathematical induction II', Journal of Mathematical Behavior 8(3), 285-304.

Durand-Guerrier, V.: 2003, 'Which notion of implication is the right one? From logical considerations to a didactic perspective', Educational Studies in Mathematics 53, 5-34. 
Edwards, L.: 1999, 'Odds and evens: Mathematical reasoning and informal proof among high school students', Journal of Mathematical Behavior, 17(4), 489-504.

Epp, S.: 1998, 'A unified framework for proof and disproof', Mathematics Teacher 9(8), 708-713.

Fischbein, E. and Kedem, I.: 1982, 'Proof and certitude in development of mathematical thinking', in A. Vermandel (ed.), Proceedings of the Sixth Annual Conference of the International Group for the Psychology of Mathematics Education, Universitaire Instelling, Antwerpen, pp. 128-131.

Fischbein, E., Tirosh, D. and Melamed, U.: 1982, 'Is it possible to measure the intuitive acceptance of the mathematical statement?', Educational Studies in Mathematics 12, 491-512.

Galbraith, P.L.: 1981, 'Aspects of proving: A critical investigation of process', Educational Studies in Mathematics 12, 1-29.

Gardiner, T. and Moreira, C.: 1999, 'Proof matters', Mathematics Teaching 88(5), 412417.

Girotto, V., Light, P. and Colbourn, C.: 1988, 'Pragmatic schemas and conditional reasoning in children', The Quarterly Journal of Experimental Psychology 40A(3), $469-482$.

Goetting, M.: 1995, The College Students' Understanding of Mathematical Proof, Doctoral Dissertation, University of Maryland.

Harel, G. and Sowder, L.: 1998, 'Students' proof schemes: Results from exploratory studies', in A.H. Schoenfeld, J. Kaput and E. Dubinsky (eds.), Research in Collegiate Mathematics Education. III, American Mathematical Society, Providence, RI, pp. 234-283.

Healy, L. and Hoyles, C.: 2000, 'A study of proof conceptions in algebra', Journal for Research in Mathematics Education 31(4), 396-428.

Hoyles, C. and Küchemann, D.: 2002, 'Students' understanding of logical implication', Educational Studies in Mathematics 51, 193-223.

Johnson-Laird, P.N., Legrenzi, P. and Sonino-Legrenzi, M.: 1972, 'Reasoning and a sense of reality', British Journal of Psychology 63(3), 395-400.

Knuth, E.J.: 1999, The Nature of Secondary School Mathematics Teachers' Conceptions of Proof, Doctoral Dissertation, University of Colorado, Boulder.

Knuth, E.J.: 2002, 'Secondary school mathematics teachers' conceptions of proof', Journal for Research in Mathematics Education 33(5), 379-405.

Knuth, E.J., Choppin, J., Slaughter, M. and Sutherland, J.: 2002, 'Mapping the conceptual terrain of middle school students' competencies in justifying and proving', in D.S. Mewborn, P. Sztajn, D.Y. White, H.G. Weigel, R.L. Bryant and K. Nooney (eds.), Proceedings of the $24^{\text {th }}$ Annual Meeting of the North American Chapter of the International Group for the Psychology of Mathematics Education, Vol. 4, Athens, GA, pp. 1693-1670.

Lee, L. and Wheeler, D.: 1987, Algebraic Thinking in High School Students: Their Conceptions of Generalization and Justification (Research Report), Department of Mathematics, Concordia University, Canada.

Leinhardt, G., Zaslavsky, O. and Stein, M.: 1990, 'Functions, graphs, and graphing: Tasks, learning, and teaching', Review of Educational Research 60(1), 1-64.

Maher, C. and Martino, A.: 1996, 'The development of the idea of mathematical proof: A 5-year case study', Journal for Research in Mathematics Education 20(1), 41-51.

Markel, W.D.: 1994, 'The role of proof in Mathematics Education', School Science and Mathematics 94(6), 291-295. 
Markovits, H. and Vachon, R.: 1989, 'Reasoning with contrary-to-fact propositions', Journal of Experimental Child Psychology 47, 398-412.

Martin, W.G. and Harel, G.: 1989, 'Proof frames of preservice elementary teachers', Journal for Research in Mathematics Education 20, 41-51.

Merriam, S.B.: 1988, Case Study Research in Education: A Qualitative Approach, JosseyBass, San Francisco.

Moore, R.: 1994, 'Making the transition to formal proof', Educational Studies in Mathematics 27, 249-266.

Morris, A.K.: 2002, 'Mathematical reasoning: adults' ability to make inductive-deductive distinction', Cognition and Instruction 20(1), 79-118.

Morris, A.K. and Sloutsky, V.: 1998, 'Understanding of logical necessity: Developmental antecedents and cognitive consequences', Child Development 69, 721-741.

Moshman, D. and Franks, B.A.: 1986, 'Development of the concept of inferential validity', Child Development 57, 153-165.

Movshovitz-Hadar, N.: 1993, 'The false coin problem, mathematical induction and knowledge fragility', Journal of Mathematical Behavior 12(3), 253-268.

National Council of Teachers of Mathematics: 2000, Principles and Standards for School Mathematics, Reston, VA.

O'Brien, T.C.: 1972, 'Logical thinking in adolescents', Educational Studies in Mathematics 4, 401-428.

O'Brien, T.C.: 1975, 'Deformation and the four card problem', Educational Studies in Mathematics 6, 23-39.

O'Brien, T.C., Shapiro, B.J. and Reali, N.: 1971, 'Logical thinking - Language and context', Educational Studies in Mathematics 4, 201-219.

Patton, M.Q.: 1990, Qualitative Evaluation and Research Methods, Sage, Newbury Park, CA.

Papanastasiou, C. and Papanastasiou, E.: 1997, 'Factors that influence students to become teachers', Educational Research and Evaluation (An International Journal on Theory and Practice) 3(4), 305-316.

Papanastasiou, C. and Papanastasiou, E.: 1998, 'What influences students to choose the elementary education major: The case of Cyprus', Mediterranean Journal of Educational Studies 3(1), 35-45.

Philippou, G.N. and Christou, C.: 1998, 'The effects of a preparatory mathematics program in changing prospective teachers' attitudes toward mathematics', Educational Studies in Mathematics 35, 189-206.

Porteous, K.: 1990, 'What do children really believe?', Educational Studies in Mathematics 21, 589-598.

Recio, A.M. and Godino, J.D.: 2001, 'Institutional and personal meanings of mathematical proof', Educational Studies in Mathematics 48, 83-99.

Reid, D.: 2002, 'Conjectures and refutations in grade 5 mathematics', Journal for Research in Mathematics Education 33(1), 5-29.

Schoenfeld, A.H.: 1986, 'On having and using geometric knowledge', in J. Hiebert (ed.), Conceptual and Procedural Knowledge: The Case of Mathematics, Lawrence Erlbaum Associates, Hillsdale, NJ, pp. 225-264.

Scholnick, E.K. and Wing, C.S.: 1988, 'Knowing when you don't know: Developmental and situational considerations', Developmental Psychology 24, 190-196.

Silver, E.A. and Carpenter, T.P.: 1989, 'Mathematics methods', in M.M. Lindquist (ed.), Fourth Mathematics Assessment of the National Educational Progress, National Council of Teachers of Mathematics, Reston, VA, pp. 10-18. 
Silver, E.A., Alacaci, C. and Stylianou, D.: 2000, 'Students' performance on extended constructed-response tasks', in P.A. Kenney and E.A. Silver (eds.), Results from the Seventh Mathematics Assessment of the National Assessment of Educational Progress, National Council of Teachers of Mathematics, Reston, VA, pp. 301-341.

Silver, J.A.: 1998, 'Can computers be used to teach proofs?', Mathematics Teacher 91(8), 660-663.

Simon, M.A. and Blume, G.W.: 1996, 'Justification in the mathematics classroom: A study of prospective elementary teachers', Journal of Mathematical Behavior 15, 3-31.

Stylianides, A.J., Stylianides, G.J. and Philippou, G.N.: 2002, 'University students' conceptions of empirical proof and proof by counterexample', in M. Tzekaki (ed.), Proceedings of the $5^{\text {th }}$ Panellenian Conference on "Didactics of Mathematics and Computers in Education”, Aristotle University of Thessaloniki, Thesalloniki, Greece, pp. 277-282.

Szombathelyi, A. and Szarvas, T.: 1998, 'Ideas for developing students' reasoning: A Hungarian perspective', Mathematics Teacher 91(8), 677-681.

Tall, D.: 1979, 'Cognitive aspects of proof, with special reference to the irrationality of $\sqrt{ } 2$ ', Proceedings of the Third International Conference for the Psychology of Mathematics Education, Warwick, pp. 203-205.

Vinner, S.: 1983, 'The notion of proof: Some aspects of students' view at the senior high level', in R. Hershkowitz (ed.), Proceedings of the Seventh International Conference for the Psychology of Mathematics Education, Rehovot, Israel, pp. 289-294.

Ward, S.L. and Overton, W.F.: 1990, 'Semantic familiarity, relevance, and the development of deductive reasoning', Developmental Psychology 26, 488-493.

Williams, E.: 1979, An Investigation of Senior High School Students' Understanding of the Nature of Mathematical Proof, Doctoral Dissertation, University of Alberta, Edmonton, Canada.

Winicki-Landman, G.: 1998, 'On proofs and their performance as works of art', Mathematics Teacher 91(8), 722-725.

Yackel, E. and Hanna, G.: 2003, 'Reasoning and proof', in J. Kilpatrick, W.G. Martin and D. Schifter (eds.), A Research Companion to Principles and Standards for School Mathematics, National Council of Teachers of Mathematics, Reston, VA, pp. 227-236.

Zack, V.: 1997, ' "You have to prove us wrong": Proof at the elementary school level', in E. Pehkonen (ed.), Proceedings of the Twenty-First International Conference on the Psychology of Mathematics Education, Vol. 4, University of Helsinki, Finland, pp. 291-298.

${ }^{1}$ The University of Michigan,

School of Education,

610 East University Avenue,

Ann Arbor, MI 48109-1259, USA,

E-mails: astylian@umich.edu,gstylian@umich.edu

${ }^{2}$ University of Cyprus,

Department of Education,

P.O. Box 20537,

1678 Nicosia, Cyprus,

E-mail: edphilip@ucy.ac.cy 